\title{
Accelerated Particle Swarm Optimizer for Optimizing Problems of Structural Engineering
}

\author{
Meenu Sharma (Dwivedi) ${ }^{1}$, Shahnwaz Alam² and M. I. Khan ${ }^{3}$
}

1,2 and 3. Department of Mechanical Engineering, Integral University, Lucknow, India (U.P.), India.

\section{Publication Info}

\section{Article history :}

Received : $03^{\text {rd }}$ May, 2018

Accepted : $7^{\text {th }}$ June, 2018

DOI : 10.18090/samriddhi.v.10i01.7

Keywords: Engineering problems, Constrained Optimization, Welded Beam Design, Structural Engineering Design, Weld bead geometry, Accelerated Particle Swarm Optimization, Design of pressure vessel, Design of the Tension, Welding parameters optimization.

*Corresponding author :

Meenu Sharma (Dwivedi)

e-mail : meenuranjan13@gmail.com

\begin{abstract}
The aim of the present work is to find a solution to non-linear constrained optimization problems of structure. Constrained optimization difficulties are practical shortcomings. The loopholes of traditional numerical methods are being removed by heuristic methods as no requirement of the functional derivatives is desired and approaches to the global way out. This article presents a "penalty guided Accelerated Particle Swarm Optimization (APSO) algorithm" to search the problem's optimal solution in the feasible region of whole search domain. There is numerical result and comparison of the structural design optimization problems. The way out by the current perspective proves to be the better than other techniques and it can be said that our findings show better solutions to engineering problems than those earlier obtained using current algorithms.
\end{abstract}

\section{INTRODUCTION}

$\mathrm{P}$ roblems based on structural engineering are extremely nonlinear, employing mixed design variables under complex restraints, where any other way rendered by calculus becomes inconsistent [13]. Design optimization is the technique to discover the most favorable parameters, to have maxima minima of desired function, subjected to constraints. Such problem of optimization is given the name of constrained optimization problems or nonlinear programming problems.

Efficacy of restrained optimization algorithms is that it unveils the optimization problems related to engineering design. These nonlinear engineering problems have been explored by several investigators that used individual aaproaches to solve them: Branch and Bound using Sequential
Linearization Algorithm [38], SQP [33], Recursive Quadratic Programming [37], Integer discrete continuous nonlinear Programming [39], Nonlinear mixed discrete Programming [1]. Problems generally have miscellaneous (e.g., continuous and discrete) input variables, nonlinear desired functions and nonlinear restraints. Restraints are well known in engineering design problems as without them the problem becomes very hard to solve.

A recent bio inspired metaheuristic is Accelerated Particle Swarm Optimization (APSO) which finds its way in highly competitive different types of problems. Moreover, a very little importance has been given to this technique in structural problems in comparison to other areas. The way to manage restraints, discussed in this work 
Yang and gave better results in knowledge till now. This compels us to use the appropriate for comparison of the utility of said technique ( results are summarised and noted later).

\section{ENGINEERING OPTIMIZATION MECHANISM}

\subsection{Constraint Handling Method}

Due to presence of both inequalities it is not accessible for solution. Despite the popularity of penalty functions, they have shortcomings out of which the main one is necessity of having large number of parameters to be adjusted and the search for the equability of the objective and penalty functions is difficult. The finding process is considerably at low pace devoid of expected results. In order to eradicate this problem, these algorithms using concept of parameter free penalty functions can be expressed as

$$
F(x)= \begin{cases}f(x) & \text { if } x \in S \\ f_{w}+\sum_{z=1}^{p+q} g_{z}(x) & \text { if } x \notin S\end{cases}
$$

where $x$ are solutions and $\mathrm{f} 34 \mathrm{w}$ is the least desired solution.

\subsection{Structural Design Optimization}

Mechanical design optimization problems can be formulated as a nonlinear programming (NLP) problem. Unlike generic NLP problems which only contain continuous or integer variables, mechanical design optimizations usually involve continuous, binary, discrete and integer variables. The binary variables are usually deployed to formulate the design problem to select alternative options. The discrete variables represent standardization constraints such as the diameters of standard sized bolts. Integer variables usually occur when the numbers of objects are design variables, such as the number of gear teeth. Adopting the different variables can be formulised as

$$
\begin{aligned}
\text { Minimize } & f(x) \\
\text { subject to } & h_{k}(x)=0 \quad ; \quad k=1,2, \ldots, p \\
& g_{j}(x) \leq 0 \quad ; \quad j=1,2, \ldots, q \\
& l_{i} \leq x_{i} \leq u_{i} \quad ; \quad i=1,2, \ldots, n
\end{aligned}
$$

where $\mathrm{x}=[\mathrm{x} 1, \mathrm{x} 2, \ldots, \mathrm{xn}] \mathrm{T}$ denotes the decision solution vectors; $\mathrm{f}$ is the objective function; $\mathrm{li}$ and ui are the minimum and maximum permissible values for the ith variable respectively; $p$ is equality number and $q$ is the number of inequality constraints. Let $S=\{x \mid g z(x)\}$ or $=0, z=1,2$, $\mathrm{p}+\mathrm{q}, \mathrm{li}<\mathrm{xi}>\mathrm{ui}\}$ accessible solution and gz be the set of equalities and inequalities constraints.

\section{ACCELERATED PARTICLE SWARM OPTIMIZATION (APSO)}

\subsection{PSO}

There are lots of PSO variants and hybrid algorithms by combining PSO with popular combination of variants with other opted procedure. Trajectories of particles are examined by the technique.

The swarming particle's advancement consists of twice greater parts: a stochastic component and a deterministic component. g_ gives best value and

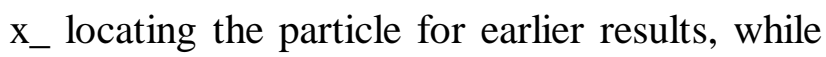
simultaneously it has a tendency to move randomly.

Let $\mathrm{xi}$ and vi be the position vector and velocity for particle i, respectively. Vector is expressed as

$$
\mathbf{v}_{i}^{t+1}=\mathbf{v}_{i}^{t}+\alpha \epsilon_{1}\left[\mathbf{g}^{*}-\mathbf{x}_{i}^{t}\right]+\beta \epsilon_{2}\left[\mathbf{x}_{i}^{*}-\mathbf{x}_{i}^{t}\right] .
$$

where Q1 and Q2 where vectorial values are between 0 and 1 . Thenwe can take, say, $\alpha \approx \beta \approx 2$.

\subsection{Accelerated PSO}

Xin-She Yang at Cambridge University in 2008 put forward APSO which is metaheuristic algorithm. Xin-She Yang, Nature Inspired Metaheuristic Algorithms, First Edition, Luniver Press, (2008) and the second edition (2010) and its. It was Nature Inspired Metaheuristic Algorithms. 
Table-3: Obtained Result (of a welded beam) using Accelerated PSO for E01

\begin{tabular}{|c|c|c|c|}
\hline \multicolumn{4}{|c|}{ DESIGN PARAMETER of E01 } \\
\hline $\mathrm{x} 1$ & $\mathrm{x} 2$ & $\mathrm{x} 3$ & $\mathrm{x} 4$ \\
\hline Best estimates: gbest=1.0196 & 9.9195 & 6.1517 & 0.68812 iteration $=1$ \\
\hline Best estimates: gbest $=1.0196$ & 9.9195 & & 0.68812 iteration $=2$ \\
\hline Best estimates: gbest $=0.4388$ & 10 & 6.75007 & 0.445817 iteration $=3$ \\
\hline Best estimates: gbest $=0.4388$ & 10 & 6.75007 & 0.445817 iteration $=4$ \\
\hline Best estimates: gbest $=0.23248$ & 9.0743 & 8.6722 & 0.3553 iteration $=5$ \\
\hline Best estimates: gbest $=0.33435$ & 6.4459 & 6.5906 & 0.41839 iteration $=6$ \\
\hline Best estimates: gbest $=0.33435$ & 6.4459 & 6.5906 & 339 iteration $=7$ \\
\hline timates: gbest $=0.33435$ & 6.4459 & 6.5906 & 339 iteration $=8$ \\
\hline mates: gbest $=0.33435$ & 6.4459 & 6.5906 & 0.41839 iteration $=9$ \\
\hline gbest $=0.21293$ & 7.5447 & 6.3711 & 0.4582 iteration $=10$ \\
\hline gbest $=0.21293$ & 7.5447 & 6.3711 & 0.4582 iteration $=11$ \\
\hline gbest $=0.21293$ & 7.5447 & 6.3711 & 0.4582 iteration $=12$ \\
\hline 0.21174 & 6.9496 & & 04 iteration $=13$ \\
\hline 0.19646 & 6.3427 & 7.7966 & 85 iteration $=14$ \\
\hline$=0.19646$ & 6.3427 & 7.7966 & 285 iteration $=15$ \\
\hline est $=0.19646$ & 6.3427 & 7.7966 & 0.28285 iteration $=16$ \\
\hline gbest $=0.19646$ & 6.3427 & 7.7966 & 0.28285 iteration $=17$ \\
\hline 0.19646 & & & 85 iteration $=18$ \\
\hline .19646 & 6.3427 & 7.7966 & 85 iteration $=19$ \\
\hline mates: gbest $=0.19646$ & 6.3427 & 7.7966 & 285 iteration $=20$ \\
\hline gbest $=0.19646$ & 6.3427 & 7.7966 & 0.28285 iteration $=21$ \\
\hline$t=0.19646$ & 6.3427 & 7.7966 & 0.28285 iteration $=22$ \\
\hline$=0.14327$ & 7.0653 & 7.95 & 0.27325 iteration $=23$ \\
\hline 0.14327 & & & 25 iteration $=24$ \\
\hline Best estimates: gbest $=0.19345$ & 7.0156 & 8.4464 & 0.24035 iteration $=25$ \\
\hline timates: gbest $=0.14228$ & 6.6736 & 8.9701 & 0.24445 iteration $=26$ \\
\hline ates: gbest $=0.14228$ & 6.6736 & 8.9701 & 0.24445 iteration $=27$ \\
\hline mates: gbest $=0.17801$ & 7.0016 & 8.8614 & 0.21635 iteration $=28$ \\
\hline imates: gbest $=0.17801$ & & 8.8614 & 0.21635 iteration $=29$ \\
\hline Best estimates: gbest $=0.15004$ & 6.0284 & 8.6249 & 0.22709 iteration $=30$ \\
\hline Best solution $=\mathbf{0 . 1 5 0 0}$ & 8.6249 & 0.2271 & $\mathrm{fmin}=\mathbf{2 . 0 3 7 2}$ \\
\hline
\end{tabular}

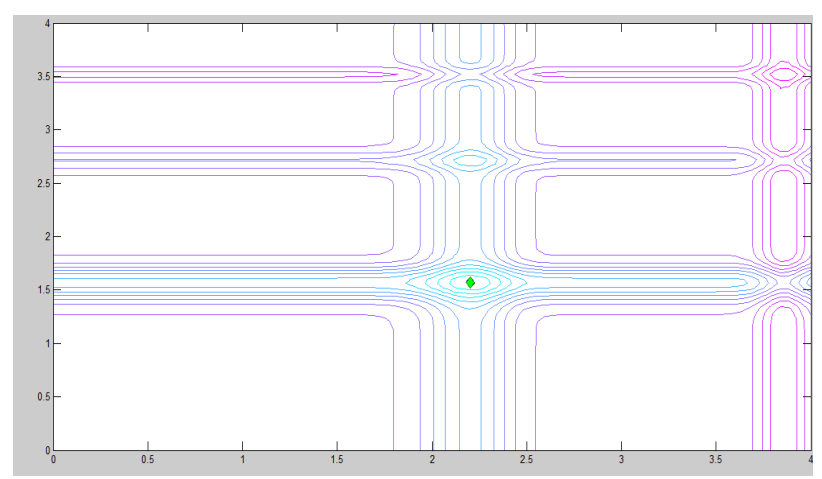

Fig.1:Direction of all roaming particles

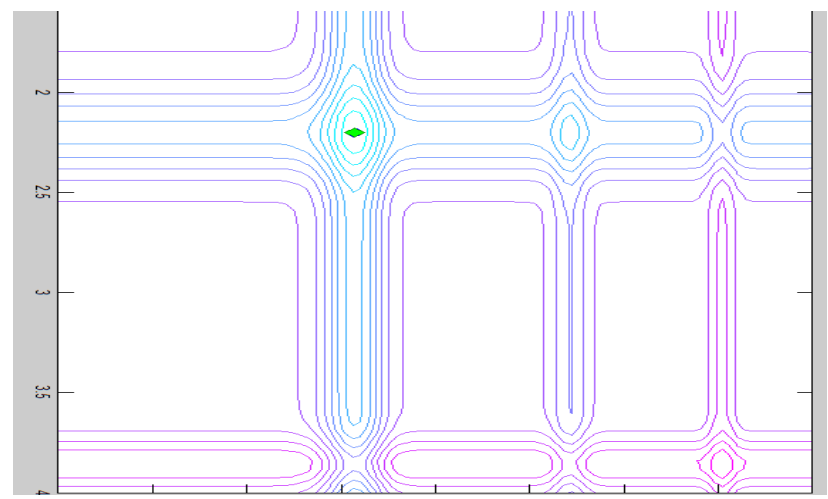

Fig.2: Trace the paths of all roaming particles
Table-1: Table 1: Best results obtained by APSO, COPSO and Mezura

\begin{tabular}{|c|c|c|c|c|}
\hline PROBLES & OPTIMAL & APSO & COPSO & MEZURA \\
\hline E01 & $\mathbf{1 . 7 2 4 8 5 2}$ & 1.724852 & 1.724852 & 1.724852 \\
\hline E02 & $\mathbf{6 , 0 5 9 . 7 1 4 3 3 5}$ & $6,059.714335$ & $6,059.714335$ & $6,059.7143$ \\
\hline E03 & NA & $2,996.348165$ & $2,996.372448$ & $2,996.348094 *$ \\
\hline E04 & $\mathbf{0 . 0 1 2 6 6 5}$ & 0.012664 & 0.012665 & 0.012689 \\
\hline
\end{tabular}

Table-2: APSO, COPSO and Mezura for mean and St. Dev. Results obtained

\begin{tabular}{|c|c|c|c|c|c|c|}
\hline \multicolumn{9}{|c}{ MEAN } & \multicolumn{3}{c|}{ ST. DEV. } \\
\hline PROBLEMS & APSO & COPSO & MEZURA & APSO & COPSO & MEZURA \\
\hline E01 & $\mathbf{2 . 0 3 7 2}$ & 1.7248 & 1.7776 & $\mathbf{0 . 1 5 0 0}$ & $1.2-05$ & $8.8 \mathrm{E}-02$ \\
\hline E02 & $6,098.0498$ & $6,071.0133$ & $6,379.9380$ & 12.1725 & 15.1011 & 210.0000 \\
\hline E03 & $2,996.4084$ & $2,996.4085$ & $2,996.3480^{*}$ & 0.0000 & 0.0286 & $0.0000^{*}$ \\
\hline E04 & 0.0130 & 0.0126 & 0.0131 & $4.3 \mathrm{E}-04$ & $1.2 \mathrm{E}-06$ & $3.9 \mathrm{E}-04$ \\
\hline
\end{tabular}

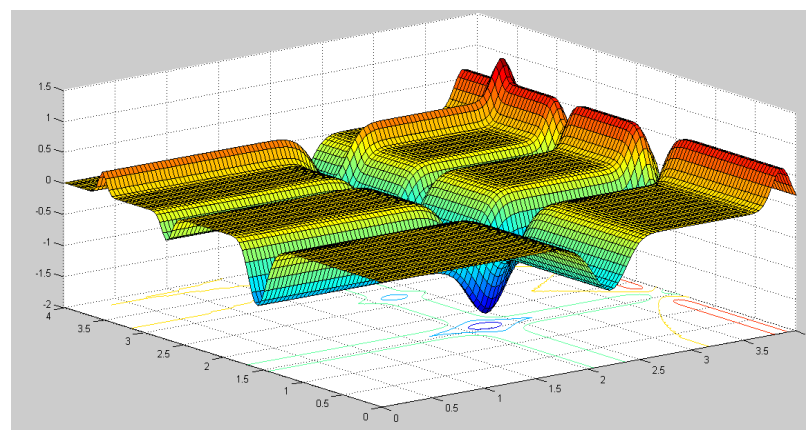

Fig.3: The shape of the function to be optimized

Hernandez Aguirre et al. and Mezura et al. Given results into the tables shown next as "COPSO" and "Mezura", respectively.

The said method used 30000 evaluations, while it is larger value. Table 1 and 2 gives the result of our work. We reference those results into the tables shown. The values obtained are shown in table 2 and 3 for E01 problem and 1 for standard APSO.

For E01. For E02, APSO gave best values and COPSO the known equivalent, but Mezura gave value upto 4 decimal points, and no report of required precision attained. For E03, APSO reached the best value, COPSO fids less accurate data, and Mezura reached an unaccessible record. APSO and COPSO managed for E04, although Mezura reported a value that is worse than the best known. For E01 and E04 low value was obtained by COPSO; for E02 and E03, our APSO found the minimum values. 


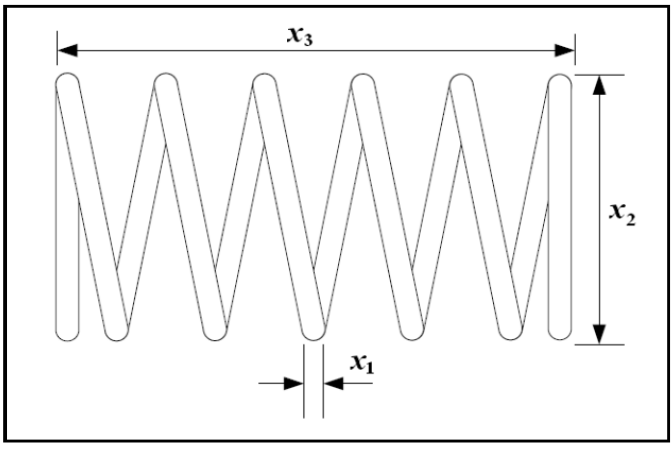

Fig.7 : Tension / Compression Spring

\section{CONCLUSIONS AND FUTURE WORK}

An Accelerated Particle Swarm Optimization (APSO) algorithm is way to deal with conditional search. The mentioned technique employs an easy way, a ring topology, with suitable formula for orientation of particle. APSO showed better technique for engineering design optimization. Comparison of the acquired results suits the desired solution. With Low objective function our method is more fruitful than any other path followed. The technique is valuable and suited for design problems. Exploring other APSO models and in performing a more detailed statistical analysis of the performance of our proposed approach is the future aspect of this finding.

\section{REFERENCES}

[1] H. Li and T. Chou. A global approach of nonlinear mixed discrete programming in design optimization. Engineering Optimization, 22:109-122, 1994.

[2] J. S. Arora, Introduction to Optimum Design, McGrawHill, New York, 1989.

[3] K. S. Lee and Z. W. Geem, A new meta-heuristic algorithm for continuous engineering optimization: harmony search theory and practice, Computer Methods in Applied Mechanics and Engineering, 194 (2005), 3902-3933.

[4] L. C. Cagnina, S. C. Esquivel and C. A. C. Coello, Solving engineering optimization problems with the simple constrained particle swarm optimizer, Informatica, 32 (2008), 319-326.
[5] C. Guo, J. Hu, B. Ye and Y. Cao. Swarm intelligence for mixed variable design optimization. Journal of Zheijiang University SCIENCE, 5(7):851-860, 1994.

[6] Yang X. S., Nature-Inspired Metaheuristic Algorithms, Luniver Press, (2008).

[7] Yang X. S., Engineering Optimization: An Introduction with Metaheuristic Applications, John Wiley and Sons, (2010).

[8] X. Hu, R. Eberhart and Y. Shi. Engineering optimization with particle swarm. 2003.

[9] E. Mezura and C. Coello. Useful Infeasible Solutions in Engineering Optimization with Evolutionary Algorithms. In Proceedings of the 4th Mexican International Conference on Artificial Intelligence, MICAI 2005. Lecture Notes on Artificial Intelligence No. 3789, pages 652-662. 2005.

[10] J. Kennedy and R. C. Eberhart, Particle swarm optimization, in: Proc. of IEEE International Conference on Neural Networks, Piscataway, NJ. pp. 1942-1948 (1995).

[11] J. Kennedy, R. C. Eberhart, Swarm intelligence, Academic Press, 2001.

[12] J. Golinski. An adaptive optimization system applied to machine synthesis. Mechanism and Machine Synthesis. 8(1973), pages 419-436, 1973.

[13] Z. Michalewicz, Genetic Algorithms + Data Structures =Evolution Programs, Springer - Verlag, Berlin, 1994.

[14] K. M. Ragsdell and D. T. Phillips, Optimal design of a class of welded structures using geometric programming, ASME Journal of Engineering for Industries, 98 (1976), 1021-1025.

[15] K. Ragsdell, and D. Phillips. Optimal design of a class of welded structures using geometric programming. ASME Journal of Engineering for Industries, 98(3):1021-1025, 1976.

[16] L. C. Cagnina, S. C. Esquivel and C. A. C. Coello, Solving engineering optimization problems with the simple constrained particle swarm optimizer, Informatica, 32 (2008), 319-326.

[17] A. H. Gandomi, X. S. Yang, and A. H. Alavi, Mixed variable structural optimization using firefly algorithm, Computers and Structures, 89 (2011a), 2325-2336. 